\title{
Lost in translation: the influence of ribosomes on bacterial mRNA decay
}

\begin{abstract}
Atilio Deana and Joel G. Belasco ${ }^{1}$ New York, New York 10016, USA

The lifetimes of bacterial mRNAs are strongly affected by their association with ribosomes. Events occurring at any stage during translation, including ribosome binding, polypeptide elongation, or translation termination, can influence the susceptibility of mRNA to ribonuclease attack. Ribosomes usually act as protective barriers that impede mRNA cleavage, but in some instances they can instead trigger the decay of the mRNA to which they are bound or send a signal that leads to widespread mRNA destabilization within a cell. The influence of translation on mRNA decay provides a quality-control mechanism for minimizing the use of poorly or improperly translated mRNAs as templates for the production of abnormal proteins that might be toxic to bacteria.
\end{abstract}

Skirball Institute of Biomolecular Medicine and Department of Microbiology, New York University School of Medicine,

Messenger RNA degradation plays a key role in controlling gene expression in all organisms. This is particularly true in bacteria, where mRNA decay rates can differ by as much as two orders of magnitude, with half-lives ranging from a fraction of a minute to more than an hour. The lifetimes of individual mRNAs are influenced by several factors, among the most important being their association with translating ribosomes. Recent discoveries have led to a better appreciation of the variety of ways in which ribosomes can impede or accelerate bacterial mRNA degradation and the regulatory significance of their ability to do so.

\section{Typical mechanism of mRNA decay in bacteria}

Bacterial RNA transcripts initially bear a triphosphate at the $5^{\prime}$ end and a stem-loop structure at the $3^{\prime}$ end. In Escherichia coli, the degradation of most mRNAs is thought to begin with internal cleavage by RNase E, an endonuclease that cuts RNA in single-stranded regions that are AU-rich (Mudd et al. 1990; Babitzke and Kushner 1991; Melefors and von Gabain 1991; Taraseviciene et al. 1991; McDowall et al. 1994). Less frequently, mRNA decay in E. coli starts with cleavage by another

[Keywords: Bacteria; mRNA degradation; mRNA half-life; prokaryotes; ribonuclease; ribosome]

${ }^{1}$ Corresponding author.

E-MAIL belasco@saturn.med.nyu.edu; FAX (212) 263-8951.

Article and publication are at http://www.genesdev.org/cgi/doi/10.1101/ gad.1348805. endonuclease, such as RNase III, RNase G, or RNase P (Schmeissner et al. 1984; Portier et al. 1987; Umitsuki et al. 2001; Li and Altman 2003). No longer protected by a 3 ' stem-loop, the resulting upstream cleavage products are promptly degraded by $3^{\prime}$ exonucleases, which can proceed all the way to the $5^{\prime}$ end unless they encounter an intercistronic stem-loop of significant thermodynamic stability. The downstream products of endonuclease cleavage also undergo rapid endonucleolytic and 3 'exonucleolytic degradation owing to their monophosphorylated 5' termini, which render them highly susceptible to further digestion due to the preference of RNase E and poly(A) polymerase (a facilitator of 3 '-exonuclease digestion) for monophosphorylated RNA substrates (Mackie 1998; Feng and Cohen 2000). 5' exonucleases are not thought to participate in bacterial mRNA degradation, as no such ribonuclease has been identified in any prokaryotic organism.

The disparate cytoplasmic lifetimes of bacterial mRNAs appear to be strongly affected by characteristics of the $5^{\prime}$-untranslated region (UTR), as evidenced by the stabilizing influence of 5 '-UTRs from long-lived mRNAs (e.g., E. coli ompA, which encodes an outer membrane protein, or Bacillus subtilis aprE, which encodes subtilisin) on more labile mRNAs to which they are fused (Belasco et al. 1986; Hambraeus et al. 2000). The key features of these $5^{\prime}$-UTRs that enable them to protect mRNA from degradation include their ribosome-binding site (RBS; see below) and the presence of a stem-loop structure at the 5' terminus (Emory et al. 1992; Arnold et al. 1998; Hambraeus et al. 2002). These findings have led to the conclusion that there is an important pathway for bacterial mRNA degradation in which bound ribosomes or base-pairing near the $5^{\prime}$ end can impede access to internal endonuclease cleavage sites by a mechanism that is poorly understood. In other cases, the degradation machinery may bypass the 5 ' end and access internal cleavage sites directly (Fig. 1; Joyce and Dreyfus 1998; Baker and Mackie 2003).

Influence of ribosome binding and translation initiation on mRNA decay

Some of the earliest evidence as to the effect of bound ribosomes on mRNA decay came from studies with an- 
Cleaved

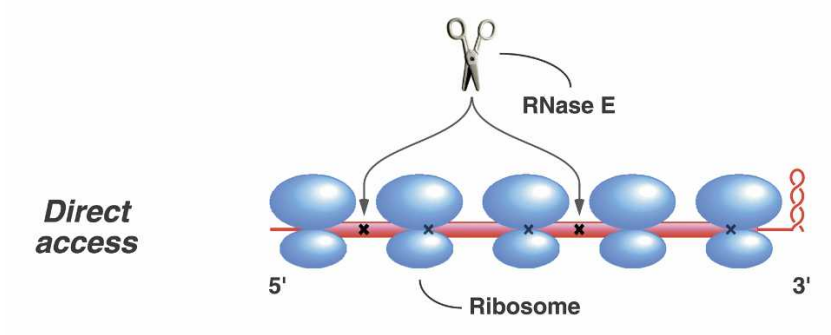

Not cleaved

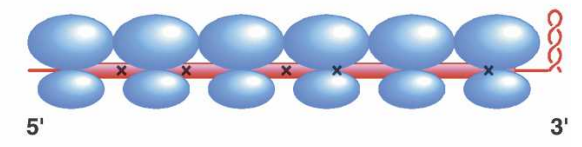

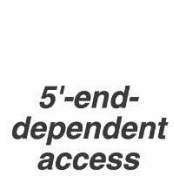
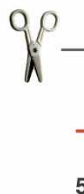

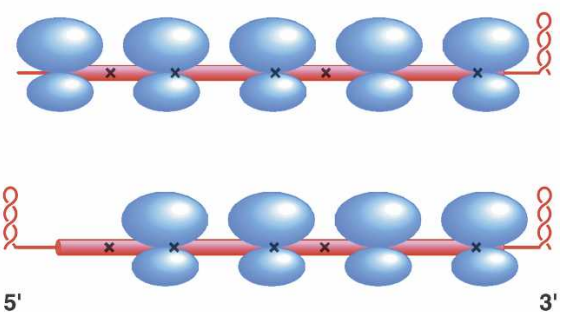

Figure 1. Pathways for the initiation of mRNA decay by RNase E and the influence of ribosomes on this event. Translated mRNA is shown undergoing the first step in degradation. (Top) Direct access. In this pathway, RNase E bypasses the 5' end and finds its way directly to internal cleavage sites within the coding and noncoding regions of the mRNA. Uninterrupted translation of the coding region by closely spaced ribosomes can mask nearby sites and help to protect them from cleavage. Although this figure depicts ribosomes as if they were static particles able to mask only the mRNA segment contacted at any particular instant, in reality each translating ribosome may be able to protect a much larger segment by transiting the coding region and dislodging RNase E from the mRNA before cleavage can occur. (Bottom) 5' -end-dependent access. In this alternative pathway, the $5^{\prime}$-terminal region of the mRNA governs the ability of RNase E to gain access to internal cleavage sites by a mechanism that is not yet understood. The presence of a ribosome at the RBS or a stem-loop at the $5^{\prime}$ end can impede such access and protect the mRNA from attack. For many transcripts, these two pathways may be able to operate concurrently, albeit at different rates; therefore, either the absence of a $5^{\prime}$-terminal barrier or the unmasking of internal sites could facilitate cleavage of such mRNAs. (Scissors) RNase E; (cylinder) protein-coding region; $(X)$ potential RNase E cleavage site. Arrows identify accessible cleavage sites.

tibiotic inhibitors of translation. However, the results of those experiments were later judged to be difficult to interpret due to indirect, cell-wide effects of these drugs unrelated to their impact on translation of the mRNAs being investigated (Lopez et al. 1998).

\section{Ribosome-binding site occupancy}

More reliable information has come from examining the influence of RBS mutations on RNA decay. For example, experiments in both E. coli and B. subtilis have shown that a variety of mRNAs can be significantly destabilized by mutations in the Shine-Dalgarno element that interfere with ribosome binding by markedly reducing its complementarity to 16S rRNA (Wagner et al. 1994; Arnold et al. 1998; Jürgen et al. 1998; Hambraeus et al. 2002; Sharp and Bechhofer 2003). In E. coli, the accelerated decay of such translationally impaired mRNAs is generally mediated by RNase E (Jain and Kleckner 1993; Arnold et al. 1998). Conversely, mutations that improve ribosome binding can prolong mRNA longevity (Jain and Kleckner 1993; Matsunaga et al. 1997). Together, these findings indicate that ribosome binding to the RBS helps to protect mRNAs from ribonuclease attack. The importance of ribosome binding for mRNA longevity probably contributes to the lability of mRNA decay intermediates that lack an RBS, a property that helps to prevent the cytoplasmic accumulation of RNA fragments.

Possible explanations for the destabilizing effect of mutations that impair ribosome binding are of two kinds (Arnold et al. 1998; Baker and Mackie 2003; Dreyfus and Joyce 2003). According to one interpretation, by directing more efficient translation initiation, a better RBS would allow closer spacing of translating ribosomes and therefore improved steric protection of potential RNase E cleavage sites in or near the protein-coding region (Fig. 1 , top). An alternative interpretation is based on evidence that mRNA features near the $5^{\prime}$ terminus can control access to internal RNase E cleavage sites; according to this interpretation, the greater ribosomal occupancy of a 5 '-proximal RBS of high affinity could impede access to such cleavage sites by blockading the $5^{\prime}$ end (Fig. 1, bottom). These two explanations are not mutually exclusive; indeed, as RNase E appears to be capable of gaining access to internal cleavage sites either directly or via the $5^{\prime}$ end, mRNAs may need both steric masking of potential cleavage sites and a $5^{\prime}$-terminal barrier to resist attack by this enzyme. In principle, the two interpretations could be distinguished by mutating the initiation codon so as to reduce the frequency of translation initiation (and thereby increase ribosomal spacing) without 
significantly diminishing the ribosome-binding affinity of the RBS and its average occupancy by ribosomes. In $E$. coli and B. subtilis, changing an initiation codon from AUG to a weaker initiation codon (GUG, CUG, or UUG) or to a codon that cannot function in translation initiation typically results in a marked reduction in translational efficiency, yet more often than not, such changes cause only a modest decrease in mRNA half-life (Wagner et al. 1994; Matsunaga et al. 1997; Arnold et al. 1998; Hambraeus et al. 2002; Sharp and Bechhofer 2003; Komarova et al. 2005). These findings suggest that a high degree of RBS occupancy by ribosomes is usually more important for mRNA longevity than is close spacing of translating ribosomes.

\section{Repression of translation by protein binding}

If a poor Shine-Dalgarno element can destabilize mRNA by impairing ribosome binding, then other impediments to ribosome binding might be expected to have a similar effect. For example, the binding of ribosomes to even a good RBS can be inhibited by competitive binding of a regulatory protein. Such inhibition is typically accompanied by a reduction in mRNA longevity. Thus, the Smlike protein $\mathrm{Hfq}$ and ribosomal protein $\mathrm{L} 1$ (the $r p l A$ gene product) each autoregulate their expression by binding their own mRNA in the vicinity of the RBS (the $h f q$ RBS or the $5^{\prime}$-proximal rplK RBS of the polycistronic rplKAJL transcript). The resulting decrease in ribosome binding causes a marked increase in the decay rate of these mRNAs (Cole and Nomura 1986; Večerek et al. 2005). Feedback regulation of the E. coli thrS (threonyl tRNA synthetase) gene has also been reported to involve coordinated effects of ThrS protein binding on translation initiation and mRNA stability (Nogueira et al. 2001).

\section{Repression of translation by base-pairing}

Ribosome binding can also be competitively inhibited via occlusion of the RBS by intra- or intermolecular basepairing, with deleterious consequences for mRNA longevity. For example, mutations outside the RBS that increase the potential for base-pairing of the RBS with the mutated RNA segment have been found to accelerate RNase E-mediated mRNA degradation by interfering with ribosome binding, whereas mutations that weaken RBS base-pairing have the opposite effect (Cho and Yanofsky 1988; Yarchuk et al. 1992). Members of a recently discovered class of cis-acting RNA regulatory elements are likely to have a similar influence on mRNA stability. These "riboswitches" each bind selectively to a small cellular metabolite, such as a vitamin or amino acid that induces a conformational change in the surrounding RNA structure. In a number of cases (e.g., the E. coli $b t u B$ and thiC mRNAs and the B. subtilis ypaA mRNA), this structural reorganization inhibits translation initiation by sequestering the RBS (Nou and Kadner 2000; Winkler et al. 2002a,b). Although not yet reported, there is every reason to expect the lifetimes of these mRNAs to be significantly diminished in the translationally repressed conformation.

Repression of translation initiation by intermolecular base-pairing can also accelerate mRNA decay. Several small untranslated RNAs (sRNAs) have been shown to control gene expression by base-pairing at or near the RBS of target mRNAs (Gottesman 2004). Many of these sRNAs mediate the regulatory influence of environmental signals such as cell density, iron starvation, temperature, oxidative stress, or phosphosugar stress, and they do so very effectively despite being imperfectly complementary to the mRNA elements with which they anneal. sRNAs are thought, in many cases, to be delivered to their mRNA targets by Hfq, whose presence can also help to protect these sRNAs from degradation (Sledjeski et al. 2001; Massé et al. 2003; Moll et al. 2003; Geissmann and Touati 2004). Two well-characterized paradigms for this important class of regulatory RNAs are RyhB, a 90-nucleotide (nt) sRNA produced when iron is scarce, and DsrA, an 87-nt sRNA produced at low temperatures. In E. coli, RyhB represses the synthesis of several proteins that store or use iron, including superoxide dismutase (the $\operatorname{sod} B$ gene product). Masking of the $\operatorname{sod} B$ RBS by intermolecular base-pairing with RyhB downregulates $\operatorname{sod} B$ translation and accelerates degradation of this mRNA by RNase E (Massé et al. 2003). Conversely, DsrA sRNA unmasks the RBS of rpoS mRNA /which encodes a stationary-phase/stress-response $\sigma$ factor) by annealing to an upstream region that otherwise occludes the RBS by intramolecular base-pairing. As a consequence of interacting with DsrA, translation of rpoS mRNA is enhanced and its lifetime is prolonged (Lease and Belfort 2000). Other sRNAs are likely to influence mRNA translation and decay via similar mechanisms.

A number of other mRNAs (e.g., the IS10 transposase and plasmid R1 repA transcripts) are perfectly complementary to the sRNAs with which they interact because both are transcribed from opposite strands of the same genetic locus. Annealing of the antisense RNA to the RBS of these mRNAs not only inhibits ribosome binding but also creates a target for mRNA cleavage by RNase III, an endonuclease that cuts long, perfectly paired RNA duplexes and certain imperfect duplexes (Blomberg et al. 1990; Case et al. 1990). In theory, the sRNA impediment to ribosome binding should alone suffice to accelerate RNase E-mediated degradation of these mRNAs even in the absence of RNase III, but this prediction has never been tested.

\section{Influence of translation elongation and termination on mRNA decay}

Even after they initiate protein synthesis, there are a variety of ways in which ribosomes can prolong or shorten mRNA lifetimes in response to events that occur during translation elongation or termination. These processes can affect the longevity of the specific subset of transcripts on which the events take place or generate 
a signal that triggers the accelerated degradation of most mRNAs within the cell.

\section{Premature translation termination}

Nonsense mutations that cause premature translation termination frequently hasten mRNA decay, especially when the premature termination codon (PTC) is close to the RBS (Nilsson et al. 1987). In E. coli, such destabilization has generally been attributed to accelerated RNase E cleavage in or near the coding region at sites (usually undefined) that normally are sterically protected by the periodic transit of translating ribosomes but are exposed when translation terminates at an upstream PTC (Arnold et al. 1998; Braun et al. 1998). Consistent with this interpretation is the finding that the rate of RNase E cleavage at a site in $r p s O$ (ribosomal protein S15) mRNA that is just downstream of the coding region depends on the distance between this site and the termination codon (Braun et al. 1998). That a PTC can also reduce the lifetime of mRNA bearing an inefficient initiation codon suggests that even infrequent traversal of the coding region by ribosomes can help to impede degradation, perhaps by dislodging RNase E from the message before cleavage can occur (Arnold et al. 1998).

Other data suggest that the destabilizing effect of PTCs cannot always be explained so simply. For example, in E. coli the marked destabilization of bla ( $\beta$ lactamase) mRNA caused by introducing a PTC near the $5^{\prime}$ end of the coding region is virtually abolished when this mutant mRNA is fused downstream of another translational unit to create a synthetic dicistronic transcript (Hansen et al. 1994). This finding suggests that the proximity of the PTC to the $5^{\prime}$-most initiation codon may in some cases be more important for rapid decay than the unmasking of potential cleavage sites downstream of the PTC. Furthermore, some transcripts (e.g., B. subtilis aprE, Rhodobacter capsulatus pufBA) are not destabilized by PTCs, presumably because other characteristics of these mRNAs or their host organisms make them resistant to ribonuclease attack under these circumstances (Klug and Cohen 1991; Hambraeus et al. 2002; Sharp and Bechhofer 2003).

\section{Uncoupling transcription and translation}

Another way that sites within the coding region can be transiently exposed to RNase E cleavage is by uncoupling transcription and translation. Normally, translating ribosomes traverse mRNA at a rate very similar to that at which it is synthesized by E. coli RNA polymerase (20-60 nt per second) (Young and Bremer 1976; Epshtein and Nudler 2003). As a result, nascent transcripts are thought to carry a full complement of ribosomes that follow closely behind the polymerase. In contrast, ribosomes cannot keep pace with T7 RNA polymerase, which synthesizes mRNA severalfold faster than the E. coli enzyme. Consequently, nascent transcripts produced by this bacteriophage enzyme in E. coli each contain a long ribosome-free segment between the lead ribosome and the transcribing polymerase, making them more susceptible to RNase E digestion by unmasking cleavage sites within the exposed segment (Iost and Dreyfus 1995). Whether this deficit of ribosomes also helps to accelerate RNase E cleavage by momentarily leaving the RBS unoccupied just after transcription begins and thereby deprotecting the $5^{\prime}$ end remains to be determined. As expected, mRNAs transcribed by $\mathrm{T} 7$ RNA polymerase acquire a normal lifetime once they are fully synthesized and the lead ribosome reaches the end of the coding region.

Interestingly, although the ribonuclease activity of RNase E is entirely contained within the $\mathrm{N}$-terminal half of the protein (McDowall and Cohen 1996), the marked instability of nascent $\mathrm{T} 7$ polymerase transcripts also requires the noncatalytic carboxyl portion of RNase E (Lopez et al. 1999). This C-terminal region contains an arginine-rich RNA-binding domain and docking sites for at least three other proteins, including a $3^{\prime}$ exoribonuclease, an RNA helicase, and a glycolytic enzyme (Taraseviciene et al. 1995; McDowall and Cohen 1996; Vanzo et al. 1998). By analogy, it seems possible that the C-terminal half of RNase E will also be found to contribute to the accelerated decay of other mRNAs that contain long segments with little or no ribosome protection, such as those that have a premature termination codon or a weak Shine-Dalgarno element.

\section{Stabilization by stalled ribosomes}

At high concentrations, antibiotics that inhibit translation elongation (e.g., chloramphenicol, tetracycline, fusidic acid) have been found to stabilize most mRNAs in bacteria. Initially, this observation was interpreted as evidence that stalled ribosomes can inhibit mRNA degradation by sterically masking mRNA cleavage sites in close proximity and/or by blockading the $5^{\prime}$ end. However, the discovery that these drugs can also prolong the lifetimes of untranslated RNAs in E. coli has raised the possibility that their stabilizing effect on mRNA may, in whole or in part, be an indirect consequence of inhibiting cellular protein synthesis (Lopez et al. 1998).

At lower concentrations insufficient to arrest protein synthesis or cause a general change in mRNA longevity, erythromycin can selectively stabilize two Staphylococcus aureus mRNAs (ermA and ermC) encoding related rRNA methyltransferases that mediate resistance to this antibiotic. In each case, a low dose of erythromycin causes ribosome stalling in a short open reading frame within the $5^{\prime}$-leader region of the mRNA. In both $S$. aureus and $B$. subtilis, the stalled ribosome induces methyltransferase synthesis by disrupting base-pairing that would otherwise occlude the RBS; in addition, it prolongs the lifetime of ermA and ermC mRNA up to 20fold by a mechanism independent of the increase in translation (Bechhofer and Dubnau 1987; Sandler and Weisblum 1988). Interestingly, when the 5' end of either erm transcript is extended by fusing additional RNA there, erythromycin-induced ribosome stalling protects 
only the mRNA segment downstream of the stall site, consistent with a directional stabilization mechanism involving $5^{\prime}$-end blockade when a ribosome is stalled nearby (Bechhofer and Zen 1989; Sandler and Weisblum 1989). A similar mechanism has been invoked to explain how the $S$. aureus cat /chloramphenicol acetyltransferase) transcript is stabilized by subinhibitory concentrations of chloramphenicol (Dreher and Matzura 1991). It remains to be determined whether mRNA stabilization by ribosomes caused by antibiotics to stall during translation elongation is a phenomenon unique to Grampositive organisms, such as $S$. aureus and B. subtilis, or common to all bacteria.

\section{Destabilization by translational pausing}

It has recently been discovered that translational pausing can sometimes induce endonucleolytic mRNA cleavage near the paused ribosome. Such cleavage has been observed in certain E. coli transcripts at sites preceded by an RNA (or nascent polypeptide) element that delays translation elongation or termination (Loomis et al. 2001; Hayes and Sauer 2003; Sunohara et al. 2004a,b). Generally, these elements end with a proline codon (or proline residue), which is followed by a stop codon in cases of delayed translation termination, but internal clusters of rare codons decoded by low-abundance aminoacyl-tRNAs (e.g., AGG codons) may have a similar effect (Sunohara et al. 2004a). Unlike mRNA cleavage triggered by ribosome pausing during translation elongation, that brought about by delayed termination appears to occur specifically within the codon at the A-site of the paused ribosome. As a result, the ribosome that induces cleavage and any ribosomes trailing it are deprived of an opportunity to terminate translation normally. Ribosome release is instead achieved by trans-translation with tmRNA, which directs translation termination after having tagged the $\mathrm{C}$ terminus of the resulting protein with an oligopeptide that mediates its swift destruction (Withey and Friedman 2003). No longer protected at its $3^{\prime}$ end by a trapped ribosome, the 5' mRNA cleavage product is expected to be rapidly degraded by $3^{\prime}$ exonucleases.

The endonuclease activity responsible for cleaving mRNA near paused ribosomes has not been identified. Experiments with ribonuclease mutants have ruled out the usual suspects (RNase E, RNase III, and RNase G) as well as several TA (toxin-antitoxin) ribonucleases (see below), raising the possibility that a previously undiscovered endonuclease or intrinsic activity of the small ribosomal subunit may be involved (Hayes and Sauer 2003; Sunohara et al. 2004a,b). In one case, an RNA helicase (HrpA) has been shown to assist cleavage near a site where ribosomes pause during elongation (Koo et al. 2004). Interestingly, though seemingly precluded from binding there until after mRNA cleavage occurs, tmRNA can facilitate (but is not required for) mRNA cleavage in the A-site of ribosomes paused at a termination codon (Sunohara et al. 2004b).
Widespread destabilization induced by amino acid starvation

Paused ribosomes not only can trigger the cleavage of mRNAs to which they are bound but also can function indirectly to induce accelerated decay of other cellular mRNAs by activating the degradative component of bacterial toxin-antitoxin systems. The E. coli chromosome encodes five such TA systems-RelE-RelB, MazF-MazE, ChpBK-ChpBI, YoeB-YefM, and YafQ-DinJ-in which the toxin is a protein capable of causing widespread mRNA degradation unless bound and inhibited by its antitoxin partner (Gerdes et al. 2005). Other bacterial genomes contain as many as 45 TA loci. Normally such toxins are maintained in an inactive state by association with their respective antitoxins, but they can be activated in cells starved for amino acids (or under other stress conditions), apparently as a result of decreased synthesis or increased degradation of the antitoxins (Aizenman et al. 1996; Christensen et al. 2001, 2003; Hazan et al. 2004). The effect on antitoxin production or turnover presumably is a direct or indirect consequence of ribosome pausing caused by a shortage of charged tRNA. Toxin activation under conditions of amino acid starvation provides a degradative mechanism for bringing about a general reduction in protein synthesis when aminoacyl-tRNAs are scarce.

Of the five E. coli toxins, three (MazF, ChpBK, and YoeB) have thus far been shown to possess intrinsic endonuclease activity that enables them, when purified, to cut RNA in the absence of ribosomes (Kamada and Hanaoka 2005; Zhang et al. 2005a,b). In bacterial cells, however, these toxins, as well as RelE, have been found to mediate cleavage predominantly in translated regions of mRNA, suggesting a role for ribosomes in helping to specify cutting sites (Christensen et al. 2003, 2004). This is clearly so for RelE-dependent mRNA cleavage, which occurs at codons positioned in the A-site of bound ribosomes (Pedersen et al. 2003). Thus, it appears that translation can influence both the activation and function of toxins encoded by TA loci.

\section{The nascent polypeptide and mRNA localization}

In addition to its direct effect on mRNA cleavage, translation can influence mRNA longevity indirectly by determining the cellular localization of transcripts. A recently discovered example of an mRNA whose localization affects its stability is the E. coli ptsG transcript, which encodes a transmembrane glucose transport protein $\left(\mathrm{IICB}^{\mathrm{Glc}}\right)$. The lifetime of ptsG mRNA is regulated by SgrS, a small untranslated RNA whose synthesis is induced by phosphosugar stress. By annealing to the ptsG RBS, SgrS inhibits translation initiation and accelerates pts $G$ mRNA degradation by RNase E (Kimata et al. 2001; Vanderpool and Gottesman 2004). Interestingly, the efficacy with which SgrS hastens the decay of pts $G$ mRNA appears to depend on the ability of translating ribosomes to bring this transcript to the cell membrane so that its protein product can be inserted cotranslation- 
ally into the lipid bilayer (Kawamoto et al. 2005). This conclusion is based on the finding that the first two transmembrane helices of either IICBGlc or another integral membrane protein must be synthesized as part of the nascent polypeptide in order for SgrS to destabilize pts $G$ mRNA.

The mechanism by which proximity to the cell membrane facilitates SgrS-mediated degradation of the $p t s G$ transcript has not been determined. In principle, this property could be explained if SgrS and/or a general degradation factor is unevenly distributed within the cytoplasm or if ribosomes compete less efficiently with SgrS for binding the RBS of polysomal transcripts that are membrane-associated. In this regard, it is noteworthy that the concentration of RNase E in E. coli appears to be highest near the membrane (Liou et al. 2001).

\section{Biological importance of linking translation and mRNA degradation}

The finding that impaired translation often accelerates bacterial mRNA decay raises important questions as to the selective evolutionary advantage conferred by rapidly degrading transcripts that are already functioning poorly, if at all, as templates for protein synthesis. One likely explanation is that this may serve as a qualitycontrol mechanism to minimize the production of aberrant proteins that might be toxic to bacterial cells. Such aberrant polypeptides could be generated from individual mRNAs either by translation termination at a premature stop codon or by adventitious translation initiation at cryptic sites within the protein-coding region; in addition, they could be produced more pervasively by decreased translational fidelity when amino acids are scarce. The potential for protein synthesis from cryptic initiation sites may be significant for poorly translated mRNAs. Such sites are likely to be widespread in the coding regions of bacterial transcripts due to flexible criteria for the sequence and location of prokaryotic translation initiation sites, but they should normally be functionally repressed by frequent translational readthrough from the principal RBS (Platt et al. 1972; Schottel et al. 1984). However, if such readthrough is infrequent because the principal RBS is inefficient or because ribosomes pause or dissociate somewhere upstream, translation from these cryptic initiation sites could become significant were the transcript not rapidly degraded.

Another potential benefit is that, whereas inhibition of translation initiation by base-pairing or protein binding is a reversible repression mechanism, RNA degradation is an irreversible process that can rid cells completely of transcripts that are no longer needed and that might otherwise impose a regulatory or energy burden. Interestingly, sRNA-mediated translational repression often triggers accelerated degradation not only of the mRNA but also of the annealed sRNA by RNase E or RNase III (Case et al. 1990; Massé et al. 2003). By coupling decay of the sRNA to the completion of its regulatory mission, cells can quickly resume translation of newly synthesized target transcripts once sRNA synthesis ceases.

The relationship between translation and mRNA decay is often reciprocal, with partial degradation altering translation in ways that facilitate the further digestion of decay intermediates. Notably, mRNA cleavage can influence translation initiation by severing or structurally reorganizing an RBS, thereby hastening additional cleavage. Furthermore, because cleavage within the coding region of a transcript can prevent translating ribosomes from encountering a termination codon, bacteria have evolved a mechanism (tmRNA-mediated trans-translation) for releasing ribosomes trapped at the $3^{\prime}$ ends of mRNA fragments that lack a stop codon. Once freed from trapped ribosomes, the $3^{\prime}$ termini of these decay intermediates can be accessed by $3^{\prime}$ exonucleases and the intermediates degraded.

\section{Outlook}

Whatever may have been the original evolutionary imperatives for linking mRNA decay and translation, it is clear that bacteria now take advantage of the connection between these two processes as a general means for controlling levels of gene expression and for adjusting those levels in response to growth conditions and environmental signals. However, the molecular mechanisms by which ribosomes influence mRNA cleavage rates are still poorly understood. Recent progress in elucidating the structure and function of bacterial ribosomes and ribonucleases makes it reasonable to expect that deeper insights into these mechanisms may soon be at hand.

\section{Acknowledgments}

We are very grateful to Marc Dreyfus for his helpful comments. The concepts presented here are based on research from many laboratories, not all of which has been mentioned because of space constraints. The writing of this review article was supported by a research grant from the National Institutes of Health (GM35769).

\section{References}

Aizenman, E., Engelberg-Kulka, H., and Glaser, G. 1996. An Escherichia coli chromosomal 'addiction module' regulated by guanosine 3',5'-bispyrophosphate: A model for programmed bacterial cell death. Proc. Natl. Acad. Sci. 93: 6059-6063.

Arnold, T.E., Yu, J., and Belasco, J.G. 1998. mRNA stabilization by the ompA 5' untranslated region: Two protective elements hinder distinct pathways for mRNA degradation. RNA 4: 319-330.

Babitzke, P. and Kushner, S.R. 1991. The Ams (altered mRNA stability) protein and ribonuclease E are encoded by the same structural gene of Escherichia coli. Proc. Natl. Acad. Sci. 88: $1-5$.

Baker, K.E. and Mackie, G.A. 2003. Ectopic RNase E sites promote bypass of $5^{\prime}$-end-dependent mRNA decay in Escherichia coli. Mol. Microbiol. 47: 75-88.

Bechhofer, D.H. and Dubnau, D. 1987. Induced mRNA stability 
in Bacillus subtilis. Proc. Natl. Acad. Sci. 84: 498-502.

Bechhofer, D.H. and Zen, K.H. 1989. Mechanism of erythromycin-induced ermC mRNA stability in Bacillus subtilis. $J$. Bacteriol. 171: 5803-5811.

Belasco, J.G., Nilsson, G., von Gabain, A., and Cohen, S.N. 1986. The stability of $E$. coli gene transcripts is dependent on determinants localized to specific mRNA segments. Cell 46: $245-251$.

Blomberg, P., Wagner, E.G., and Nordström, K. 1990. Control of replication of plasmid $\mathrm{R} 1$ : The duplex between the antisense RNA, CopA, and its target, CopT, is processed specifically in vivo and in vitro by RNase III. EMBO J. 9: 2331-2340.

Braun, F., Le Derout, J., and Régnier, P. 1998. Ribosomes inhibit an RNase E cleavage which induces the decay of the rpsO mRNA of Escherichia coli. EMBO. J. 17: 4790-4797.

Case, C.C., Simons, E.L., and Simons, R.W. 1990. The IS10 transposase mRNA is destabilized during antisense RNA control. EMBO J. 9: 1259-1266.

Cho, K.O. and Yanofsky, C. 1988. Sequence changes preceding a Shine-Dalgarno region influence $\operatorname{trp} E$ mRNA translation and decay. J. Mol. Biol. 204: 51-60.

Christensen, S.K., Mikkelsen, M., Pedersen, K., and Gerdes, K. 2001. RelE, a global inhibitor of translation, is activated during nutritional stress. Proc. Natl. Acad. Sci. 98: 14328-14333.

Christensen, S.K., Pedersen, K., Hansen, F.G., and Gerdes, K. 2003. Toxin-antitoxin loci as stress-response-elements: ChpAK/MazF and ChpBK cleave translated RNAs and are counteracted by tmRNA. J. Mol. Biol. 332: 809-819.

Christensen, S.K., Maenhaut-Michel, G., Mine, N., Gottesman, S., Gerdes, K., and Van Melderen, L. 2004. Overproduction of the Lon protease triggers inhibition of translation in Escherichia coli: Involvement of the yefM-yoeB toxin-antitoxin system. Mol. Microbiol. 51: 1705-1717.

Cole, J.R. and Nomura, M. 1986. Changes in the half-life of ribosomal protein messenger RNA caused by translational repression. J. Mol. Biol. 188: 383-392.

Dreher, J. and Matzura, H. 1991. Chloramphenicol-induced stabilization of cat messenger RNA in Bacillus subtilis. Mol. Microbiol. 5: 3025-3034.

Dreyfus, M. and Joyce, S. 2003. The interplay between translation and mRNA decay in prokaryotes. In Translation mechanisms (eds. J. Lapointe and L. Brakier-Gingras), pp. 165-183. Landes Bioscience, Georgetown, TX.

Emory, S.A., Bouvet, P., and Belasco, J.G. 1992. A 5'-terminal stem-loop structure can stabilize mRNA in Escherichia coli. Genes \& Dev. 6: 135-148.

Epshtein, V. and Nudler, E. 2003. Cooperation between RNA polymerase molecules in transcription elongation. Science 300: 801-805.

Feng, Y. and Cohen, S.N. 2000. Unpaired terminal nucleotides and $5^{\prime}$ monophosphorylation govern 3 ' polyadenylation by Escherichia coli poly(A) polymerase I. Proc. Natl. Acad. Sci. 97: 6415-6420.

Geissmann, T.A. and Touati, D. 2004. Hfq, a new chaperoning role: Binding to messenger RNA determines access for small RNA regulator. EMBO T. 23: 396-405.

Gerdes, K., Christensen, S.K., and Løbner-Olesen, A. 2005. Prokaryotic toxin-antitoxin stress response loci. Nat. Rev. Microbiol. 3: 371-382.

Gottesman, S. 2004. The small RNA regulators of Escherichia coli: Roles and mechanisms. Annu. Rev. Microbiol. 58: 303328.

Hambraeus, G., Persson, M., and Rutberg, B. 2000. The aprE leader is a determinant of extreme mRNA stability in Bacillus subtilis. Microbiology 146: 3051-3059.

Hambraeus, G., Karhumaa, K., and Rutberg, B. 2002. A 5' stem- loop and ribosome binding but not translation are important for the stability of Bacillus subtilis aprE leader mRNA. Microbiology 148: 1795-1803.

Hansen, M.J., Chen, L.-H., Fejzo, M.L.S., and Belasco, J.G. 1994. The ompA 5' untranslated region impedes a major pathway for mRNA degradation in Escherichia coli. Mol. Microbiol. 12: 707-716.

Hayes, C.S. and Sauer, R.T. 2003. Cleavage of the A site mRNA codon during ribosome pausing provides a mechanism for translational quality control. Mol. Cell 12: 903-911.

Hazan, R., Sat, B., and Engelberg-Kulka, H. 2004. Escherichia coli mazEF-mediated cell death is triggered by various stressful conditions. J. Bacteriol. 186: 3663-3669.

Iost, I. and Dreyfus, M. 1995. The stability of Escherichia coli lacZ mRNA depends upon the simultaneity of its synthesis and translation. EMBO J. 14: 3252-3261.

Jain, C. and Kleckner, N. 1993. IS10 mRNA stability and steady state levels in Escherichia coli: Indirect effects of translation and role of rne function. Mol. Microbiol. 9: 233-247.

Joyce, S.A. and Dreyfus, M. 1998. In the absence of translation, RNase E can bypass 5' mRNA stabilizers in Escherichia coli. J. Mol. Biol. 282: 241-254.

Jürgen, B., Schweder, T., and Hecker, M. 1998. The stability of mRNA from the gsiB gene of Bacillus subtilis is dependent on the presence of a strong ribosome binding site. Mol. Gen. Genet. 258: 538-545.

Kamada, K. and Hanaoka. F. 2005. Conformational change in the catalytic site of the ribonuclease YoeB toxin by YefM antitoxin. Mol. Cell 19: 497-509.

Kawamoto, H., Morita, T., Shimizu, A., Inada, T., and Aiba, H. 2005. Implication of membrane localization of target mRNA in the action of a small RNA: Mechanism of post-transcriptional regulation of glucose transporter in Escherichia coli. Genes \& Dev. 19: 328-338.

Kimata, K., Tanaka, Y., Inada, T., and Aiba, H. 2001. Expression of the glucose transporter gene, $p t s G$, is regulated at the mRNA degradation step in response to glycolytic flux in Escherichia coli. EMBO I. 20: 3587-3595.

Klug, G. and Cohen, S.N. 1991. Effects of translation on degradation of mRNA segments transcribed from the polycistronic puf operon of Rhodobacter capsulatus. I. Bacteriol. 173: $1478-1484$

Komarova, A.V., Tchufistova, L.S., Dreyfus, M., and Boni, I.V. 2005. AU-rich sequences within $5^{\prime}$ untranslated leaders enhance translation and stabilize mRNA in Escherichia coli. J. Bacteriol. 187: 1344-1349.

Koo, J.T., Choe, J., and Moseley, S.L. 2004. HrpA, a DEAH-box RNA helicase, is involved in mRNA processing of a fimbrial operon in Escherichia coli. Mol. Microbiol. 52: 1813-1826.

Lease, R.A. and Belfort, M. 2000. A trans-acting RNA as a control switch in Escherichia coli: DsrA modulates function by forming alternative structures. Proc. Natl. Acad. Sci. 97: 9919-9924.

Li, Y. and Altman, S. 2003. A specific endoribonuclease, RNase $\mathrm{P}$, affects gene expression of polycistronic operon mRNAs. Proc. Natl. Acad. Sci. 100: 13213-13218.

Liou, G.G., Jane, W.N., Cohen, S.N., Lin, N.S., and Lin-Chao, S. 2001. RNA degradosomes exist in vivo in Escherichia coli as multicomponent complexes associated with the cytoplasmic membrane via the $\mathrm{N}$-terminal region of ribonuclease $\mathrm{E}$. Proc. Nat1. Acad. Sci. 98: 63-68.

Loomis, W.P., Koo, J.T., Cheung, T.P., and Moseley, S.L. 2001. A tripeptide sequence within the nascent DaaP protein is required for mRNA processing of a fimbrial operon in Escherichia coli. Mol. Microbiol. 39: 693-707.

Lopez, P.J., Marchand, I., Yarchuk, O., and Dreyfus, M. 1998. 
Translation inhibitors stabilize Escherichia coli mRNAs independently of ribosome protection. Proc. Natl. Acad. Sci. 95: 6067-6072.

Lopez, P.J., Marchand, I., Joyce, S.A., and Dreyfus, M. 1999. The C-terminal half of RNase E, which organizes the Escherichia coli degradosome, participates in mRNA degradation but not rRNA processing in vivo. Mol. Microbiol. 33: 188-199.

Mackie, G.A. 1998. Ribonuclease $\mathrm{E}$ is a $5^{\prime}$-end-dependent endonuclease. Nature 395: 720-723.

Massé, E., Escorcia, F.E., and Gottesman, S. 2003. Coupled degradation of a small regulatory RNA and its mRNA targets in Escherichia coli. Genes \& Dev. 17: 2374-2383.

Matsunaga, J., Simons, E.L., and Simons, R.W. 1997. Escherichia coli RNase III ( $\mathrm{nnc}$ ) autoregulation occurs independently of $r n c$ gene translation. Mol. Microbiol. 26: 1125-1135.

McDowall, K.J. and Cohen, S.N. 1996. The N-terminal domain of the rne gene product has RNase E activity and is nonoverlapping with the arginine-rich RNA-binding site. J. Mol. Biol. 255: 349-355.

McDowall, K.J., Lin-Chao, S., and Cohen, S.N. 1994. A + U content rather than a particular nucleotide order determines the specificity of RNase E cleavage. J. Biol. Chem. 269: 1079010796.

Melefors, Ö. and von Gabain, A. 1991. Genetic studies of cleavage-initiated mRNA decay and processing of ribosomal 9S RNA show that the Escherichia coli ams and rne loci are the same. Mol. Microbiol. 5: 857-864.

Moll, I., Afonyushkin, T., Vytvytska, O., Kaberdin, V.R., and Bläsi, U. 2003. Coincident Hfq binding and RNase E cleavage sites on mRNA and small regulatory RNAs. RNA 9: 13081314.

Mudd, E.A., Krisch, H.M., and Higgins, C.F. 1990. RNase E, an endoribonuclease, has a general role in the chemical decay of E. coli mRNA: Evidence that rne and ams are the same genetic locus. Mol. Microbiol. 4: 2127-2135.

Nilsson, G., Belasco, J.G., Cohen, S.N., and von Gabain, A. 1987. Effect of premature termination of translation on mRNA stability depends on the site of ribosome release. Proc. Natl. Acad. Sci. 84: 4890-4894.

Nogueira, T., de Smit, M., Graffe, M., and Springer, M. 2001. The relationship between translational control and mRNA degradation for the Escherichia coli threonyl-tRNA synthetase gene. J. Mol. Biol. 310: 709-722.

Nou, X. and Kadner, R.J. 2000. Adenosylcobalamin inhibits ribosome binding to btuB RNA. Proc. Natl. Acad. Sci. 97: 7190-7195.

Pedersen, K., Zavialov, A.V., Pavlov, M.Y., Elf, J., Gerdes, K., and Ehrenberg, M. 2003. The bacterial toxin RelE displays codon-specific cleavage of mRNAs in the ribosomal A site. Cell 112: 131-140.

Platt, T., Weber, K., Ganem, D., and Miller, J.H. 1972. Translational restarts: AUG reinitiation of a lac repressor fragment. Proc. Natl. Acad. Sci. 69: 897-901.

Portier, C., Dondon, L., Grunberg-Manago, M., and Régnier, P. 1987. The first step in functional inactivation of the Escherichia coli polynucleotide phosphorylase messenger is a ribonuclease III processing at the $5^{\prime}$ end. EMBO J. 6: 21652170.

Sandler, P. and Weisblum, B. 1988. Erythromycin-induced stabilization of ermA messenger RNA in Staphylococcus aureus and Bacillus subtilis. J. Mol. Biol. 203: 905-915.

. 1989. Erythromycin-induced ribosome stall in the ermA leader: A barricade to $5^{\prime}$-to- $3^{\prime}$ nucleolytic cleavage of the ermA transcript. J. Bacteriol. 171: 6680-6688.

Schmeissner, U., McKenney, K., Rosenberg, M., and Court, D. 1984. Removal of a terminator structure by RNA processing regulates int gene expression. J. Mol. Biol. 176: 39-53.

Schottel, J.L., Sninsky, J.J., and Cohen, S.N. 1984. Effects of alterations in the translation control region on bacterial gene expression: Use of cat gene constructs transcribed from the lac promoter as a model system. Gene 28: 177-193.

Sharp, J.S. and Bechhofer, D.H. 2003. Effect of translational signals on mRNA decay in Bacillus subtilis. I. Bacteriol. 185: 5372-5379.

Sledjeski, D.D., Whitman, C., and Zhang, A. 2001. Hfq is necessary for regulation by the untranslated RNA DsrA. J. Bacteriol. 183: 1997-2005.

Sunohara, T., Jojima, K., Tagami, H., Inada, T., and Aiba, H. 2004a. Ribosome stalling during translation elongation induces cleavage of mRNA being translated in Escherichia coli. J. Biol. Chem. 279: 15368-15375.

Sunohara, T., Jojima, K., Yamamoto, Y., Inada, T., and Aiba, H. 2004b. Nascent-peptide-mediated ribosome stalling at a stop codon induces mRNA cleavage resulting in nonstop mRNA that is recognized by tmRNA. RNA 10:378-386.

Taraseviciene, L., Miczak, A., and Apirion, D. 1991. The gene specifying RNase E (rne) and a gene affecting mRNA stability (ams) are the same gene. Mol. Microbiol. 5: 851-855.

Taraseviciene, L., Björk, G.R., and Uhlin, B.E. 1995. Evidence for an RNA binding region in the Escherichia coli processing endoribonuclease RNase E. J. Biol. Chem. 270: 2639126398.

Umitsuki, G., Wachi, M., Takada, A., Hikichi, T., and Nagai, K. 2001. Involvement of RNase G in in vivo mRNA metabolism in Escherichia coli. Genes Cells 6: 403-410.

Vanderpool, C.K. and Gottesman, S. 2004. Involvement of a novel transcriptional activator and small RNA in post-transcriptional regulation of the glucose phosphoenolpyruvate phosphotransferase system. Mol. Microbiol. 54: 1076-1089.

Vanzo, N.F., Li, Y.-S., Py, B., Blum, E., Higgins, C.F., Raynal, L.C., Krisch, H.M., and Carpousis, A.J. 1998. Ribonuclease E organizes the protein interactions in the Escherichia coli RNA degradosome. Genes \& Dev. 12: 2770-2781.

Večerek, B., Moll, I., and Bläsi, U. 2005. Translational autocontrol of the Escherichia coli hf $q$ RNA chaperone gene. RNA 11: 976-984.

Wagner, L.A., Gesteland, R.F., Dayhuff, T.J., and Weiss, R.B. 1994. An efficient Shine-Dalgarno sequence but not translation is necessary for lacZ mRNA stability in Escherichia coli. J. Bacteriol. 176: 1683-1688.

Winkler, W., Nahvi, A., and Breaker, R.R. 2002a. Thiamine derivatives bind messenger RNAs directly to regulate bacterial gene expression. Nature 419: 952-956.

Winkler, W.C., Cohen-Chalamish, S., and Breaker, R.R. 2002b. An mRNA structure that controls gene expression by binding FMN. Proc. Natl. Acad. Sci. 99: 15908-15913.

Withey, J.H. and Friedman, D.I. 2003. A salvage pathway for protein structures: tmRNA and trans-translation. Annu. Rev. Microbiol. 57: 101-123.

Yarchuk, O., Jacques, N., Guillerez, J., and Dreyfus, M. 1992. Interdependence of translation, transcription and mRNA degradation in the lacZ gene. J. Mol. Biol. 226: 581-596.

Young, R. and Bremer, H. 1976. Polypeptide-chain-elongation rate in Escherichia coli $\mathrm{B} / \mathrm{r}$ as a function of growth rate. Biochem. J. 160: 185-194.

Zhang, Y., Zhang, J., Hara, H., Kato, I., and Inouye, M. 2005a. Insights into the mRNA cleavage mechanism by MazF, an mRNA interferase. J. Biol. Chem. 280: 3143-3150.

Zhang, Y., Zhu, L., Zhang, J., and Inouye, M. 2005b. Characterization of ChpBK, an mRNA interferase from Escherichia coli. J. Biol. Chem. 280: 26080-26088. 


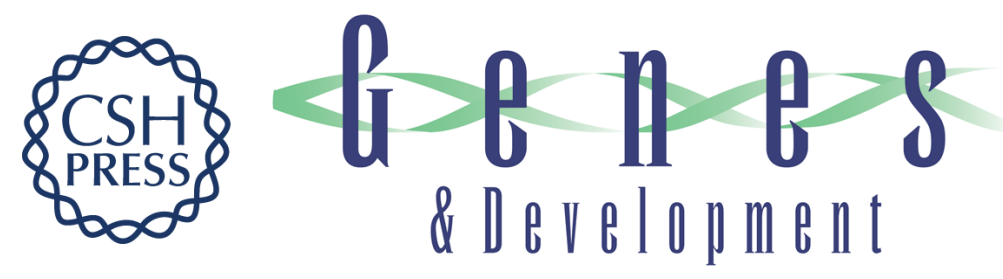

\section{Lost in translation: the influence of ribosomes on bacterial mRNA decay}

Atilio Deana and Joel G. Belasco

Genes Dev. 2005, 19:

Access the most recent version at doi:10.1101/gad.1348805

References This article cites 79 articles, 37 of which can be accessed free at: http://genesdev.cshlp.org/content/19/21/2526.full.html\#ref-list-1

License

Email Alerting

Receive free email alerts when new articles cite this article - sign up in the box at the top Service right corner of the article or click here.

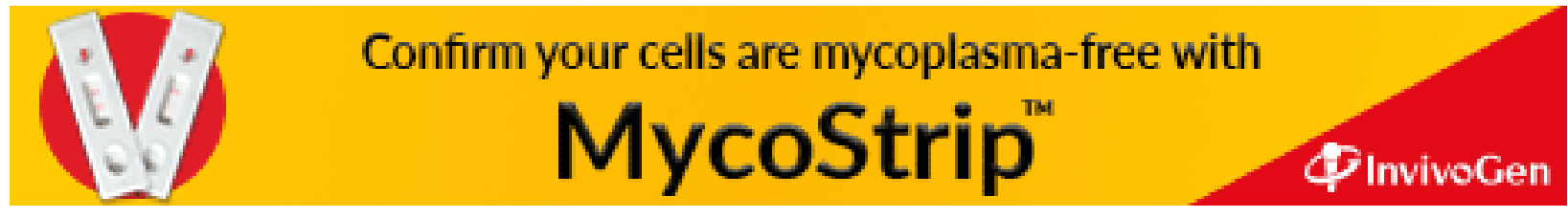

